\title{
Landauer's erasure principle in a squeezed thermal memory
}

\author{
Jan Klaers ${ }^{1}$ * \\ ${ }^{1}$ Complex Photonic Systems (COPS), MESA ${ }^{+}$Institute for Nanotechnology, \\ University of Twente, 7522 NB Enschede, The Netherlands
}

\begin{abstract}
Landauer's erasure principle states that the irreversible erasure of a one-bit memory, embedded in a thermal environment, is accompanied with a work input of at least $k_{\mathrm{B}} T \ln 2$. Fundamental to that principle is the assumption that the physical states representing the two possible logical states are close to thermal equilibrium. Here, we propose and theoretically analyze a minimalist mechanical model of a one-bit memory operating with squeezed thermal states. It is shown that the Landauer energy bound is exponentially lowered with increasing squeezing factor. Squeezed thermal states, which may naturally arise in digital electronic circuits operating in a pulse-driven fashion, thus can be exploited to reduce the fundamental energy costs of an erasure operation.
\end{abstract}

Energy dissipation is one of the main design considerations in digital electronics today [1-3]. Smaller transistors operating at lower-voltages are a natural design choice that may reduce the power consumption of central processing units. In 1961, Rolf Landauer argued that there exists a limit to which the power consumption of certain logical operations can be reduced. Landauer's principle states that the erasure (or reset) of one bit of classical information is necessarily associated with an entropy increase of at least $k_{\mathrm{B}} \ln 2$ and an energy input of at least $k_{\mathrm{B}} T \ln 2$ [411. For the present generation of silicon-based integrated circuits, the energy dissipation per logic operation is about a factor of 1000 larger than the Landauer limit. It is, however, predicted that the Landauer limit will be reached within the next decades [1 3]. Thus, improvements in our understanding of energy dissipation in information-processing devices are of both scientific interest and of technological relevance. Due to the ongoing miniaturization, non-equilibrium and quantum effects must be taken into account $[12-18$. In this work, it is theoretically demonstrated that memory devices embedded in a squeezed thermal environment are unbounded by the Landauer limit. In these environments, thermal fluctuations show fast periodic amplitude modulations, which can be exploited to reduce the minimum energy costs for an erasure operation below the standard Landauer limit. This situation may naturally occur in digital electronic circuits operating in a pulsedriven fashion and, in future, could be exploited to build more energy-efficient electronic devices.

Squeezed thermal states are the classical analog of squeezed coherent states in quantum mechanics. Both states are characterized by an asymmetric phase space density as opposed to the rotationally invariant phase space densities of coherent, thermal, or vacuum states. A mechanical oscillator may be prepared in a squeezed thermal state [19, 20] by a periodic modulation of the spring constant 21]. This leads to a state with reduced thermal fluctuations in one quadrature (e.g. momentum) and enhanced fluctuations in the orthogonal quadrature

\footnotetext{
* j.klaers@utwente.nl
}

(e.g. position). In the context of heat engines, squeezed thermal reservoirs have been proposed as a resource for work generation unbounded by the standard Carnot limit [22 26]. Due to the non-equilibrium nature of these reservoirs, this does not violate the second law of thermodynamics. In recent work [27, we have demonstrated a physical realization of such an engine, in which the working medium consists of a vibrating nano-beam that is driven by squeezed electronic noise to perform work beyond the Carnot limit. We have furthermore demonstrated that a phase-selective thermal coupling allows to extract work from a single squeezed thermal reservoir, which is not possible with a standard thermal reservoir 28.

In this work, we propose and theoretically analyze a minimalist mechanical model of a one-bit memory subject to squeezed thermal noise. This memory consists of a single particle that is trapped in a harmonic potential. The trap can be spatially divided into two halves by a partition in the trap center. If the particle resides on the left-hand side of the trap, the memory is regarded as being in the logical state ' 0 '; if it is located on the right hand side, the memory is in the ' 1 ' state. We further assume that the particle is coupled to a squeezed thermal reservoir, which can be modeled by introducing a stochastic force $f=f(t)$ to its equation of motion, as described by the Langevin equation $m \ddot{x}=F(x)-c \dot{x}+f$. Here $m$ denotes the mass of the oscillator, $c$ is the viscous damping coefficient, and $F(x)=-m \omega_{0}^{2} x$ describes the restoring force with $\omega_{0}$ as the undamped oscillator frequency. The stochastic force $f(t)$ is synthesized from two independent noise signals $\xi_{1,2}(t)$ that are mixed with sine and cosine component of a local oscillator at frequency $\omega=2 \pi \nu$ [27]:

$$
f(t)=a_{0}\left[\mathrm{e}^{+r} \xi_{1}(t) \cos (\omega t)+\mathrm{e}^{-r} \xi_{2}(t) \sin (\omega t)\right]
$$

The squeezed thermal reservoir modeled by $f(t)$ is characterized by an overall amplitude $a_{0}$ and a squeezing parameter $r$ that tunes the imbalance between the two orthogonal quadratures. Assuming $\xi_{1,2}(t)$ to be white noise, the power spectral density is frequencyindependent and increases exponentially with the squeezing factor: $\operatorname{psd}[f](\omega) \propto \cosh 2 r$. The squeezing introduces fast periodic amplitude modulations in the stochas- 
(a)

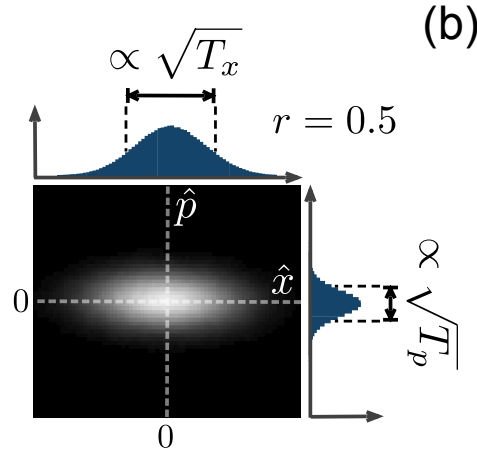

(b)

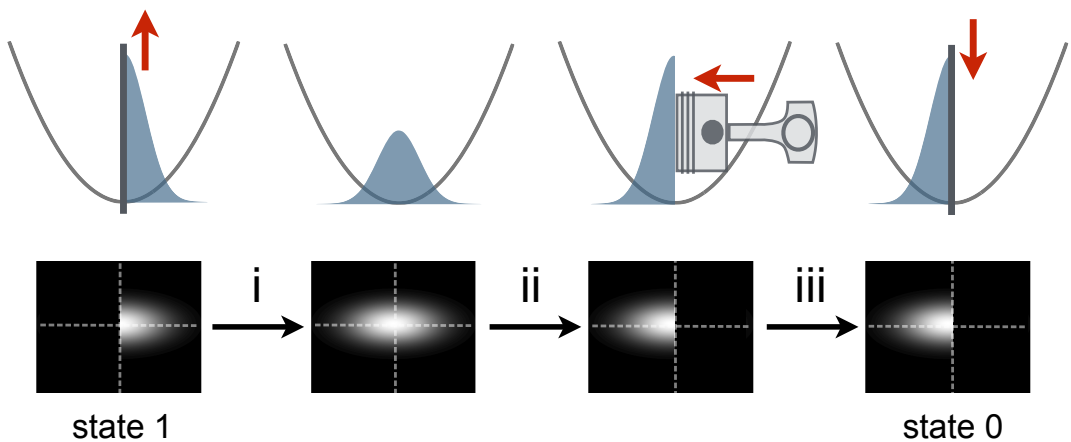

Figure 1. (a) Stroboscopic phase space probability density of a squeezed thermal state (squeezing factor $r=0.5$, relative timing $\left.t_{0}=0.24 \nu^{-1}\right)$. The thermal fluctuations in one quadrature are reduced, while the orthogonal quadrature shows increased fluctuations. The variances of the line integrated distributions (shown in blue) correspond to two temperatures $T_{x}, T_{p}$ that govern the thermal fluctuations of the system. (b) Scheme to erase one bit of information in a squeezed thermal memory: (i) removal of partition and free expansion, (ii) stroboscopic compression to half of the volume (iii) insertion of partition. During the process, the single-particle gas is assumed to be in contact with the squeezed thermal reservoir at all times.

tic force as can be seen from

$$
\left\langle f^{2}(t)\right\rangle=\frac{a_{0}^{2}}{3}\left[\mathrm{e}^{-2 r}+2 \sinh (2 r) \cos ^{2}(\omega t)\right],
$$

in which $\langle\ldots\rangle$ denotes the statistical average over many independent realizations.

The impact of the so defined stochastic force on the single-particle gas can be investigated by means of phase space densities, such as presented in Fig. 11a. The numerical results shown here, and in the rest of this work, have been obtained by integrating the Langevin equation using the Runge-Kutta method (fourth order) with constant time steps. The local oscillator in eq. (1) is assumed to be resonant with the undamped oscillator $\left(\omega=2 \pi \nu=\omega_{0}\right)$ and the noise functions $\xi_{1,2}(t) \in[-1 ; 1]$ are sampled from white noise generated by a (pseudo)random number generator with a high-frequency cutoff at $\nu / 2$. The phase space density presented in Fig. 1 a demonstrates a reduction in thermal fluctuations in the squeezed quadrature and an increase in the anti-squeezed quadrature (squeezing factor $r=0.5$ ). The quantities $\hat{x}$ and $\hat{p}$, corresponding to the two axes of the phase space plots, may be regarded as two orthogonal quadratures co-rotating with the driving force (rotating frame). Another valid interpretation is to regard $\hat{x}=x \sqrt{m \omega / \hbar}$ and $\hat{p}=p / \sqrt{\hbar \omega m}$ as dimensionless instances of the actual physical position $x$ and momentum $p$ (laboratory frame). In this case, the diagram in Fig. 17a represents a stroboscopic phase space density measured at equidistant points in time $t_{0}, \nu^{-1}+t_{0}, 2 \nu^{-1}+t_{0}, \ldots$, where $t_{0}$ sets the relative timing of the observations with respect to the local oscillator in the stochastic force. For the remainder of this work, we restrict our presentation to the laboratory frame. An important consequence is that any interaction with the system has to be performed in a stroboscopic fashion. A spatial compression, for example, needs to be divided into a sequence of smaller compression steps that have to be executed with the desired timing $t_{0}$. A concrete realization of the latter is moving the piston with the velocity $v(t)=v_{\max } \cos ^{2 n}\left(\omega\left(t-t_{0}\right)\right)$, in which $n$ is a large positive integer and the maximum velocity $v_{\max }$ is kept sufficiently small.

Squeezed thermal states can be understood in terms of a generalized Gibbs ensemble [27, 29]. The thermal fluctuations of the two orthogonal quadratures $\hat{x}$ and $\hat{p}$ are controlled by two different temperatures $T_{x}$ and $T_{p}$, which take the role of state variables (see Fig. 17). The corresponding stroboscopic phase space density follows

$$
\rho_{\mathrm{sq}}(\hat{x}, \hat{p}) \propto \exp \left(-\frac{\hbar \omega \hat{x}^{2}}{2 k_{\mathrm{B}} T_{x}}-\frac{\hbar \omega \hat{p}^{2}}{2 k_{\mathrm{B}} T_{p}}\right) .
$$

An effective system temperature $T$ may be defined as $T=\sqrt{T_{x} T_{p}}$. A consequence of this definition is that an isothermal squeezing operation $(T=$ const) does not increase the entropy of the state [27].

The scheme to erase one bit of information in a squeezed thermal memory is shown in Fig. 1 p. During the process, the single-particle gas is assumed to be in contact with the squeezed thermal reservoir at all times. First, the partition is removed and the gas expands freely (step i in Fig. 1). In the second step (ii), the gas is compressed by a piston. In the last step (iii), the partition is put back in the center of the trap. This procedure initializes the memory in the state 0 regardless of the initial conditions. The general idea behind the scheme is to use squeezing as a means to reduce the occurrence of large positive momenta at the position of the piston during the compressions steps. The latter reduces the pressure and, thus, the work required for the compression. In our analysis, we make several assumptions: it is assumed that the removal (and insertion) of the partition is free of any energy cost. The collisions of the particle with the piston are considered fully elastic. We also assume that the collisions leave the motional state of the piston 

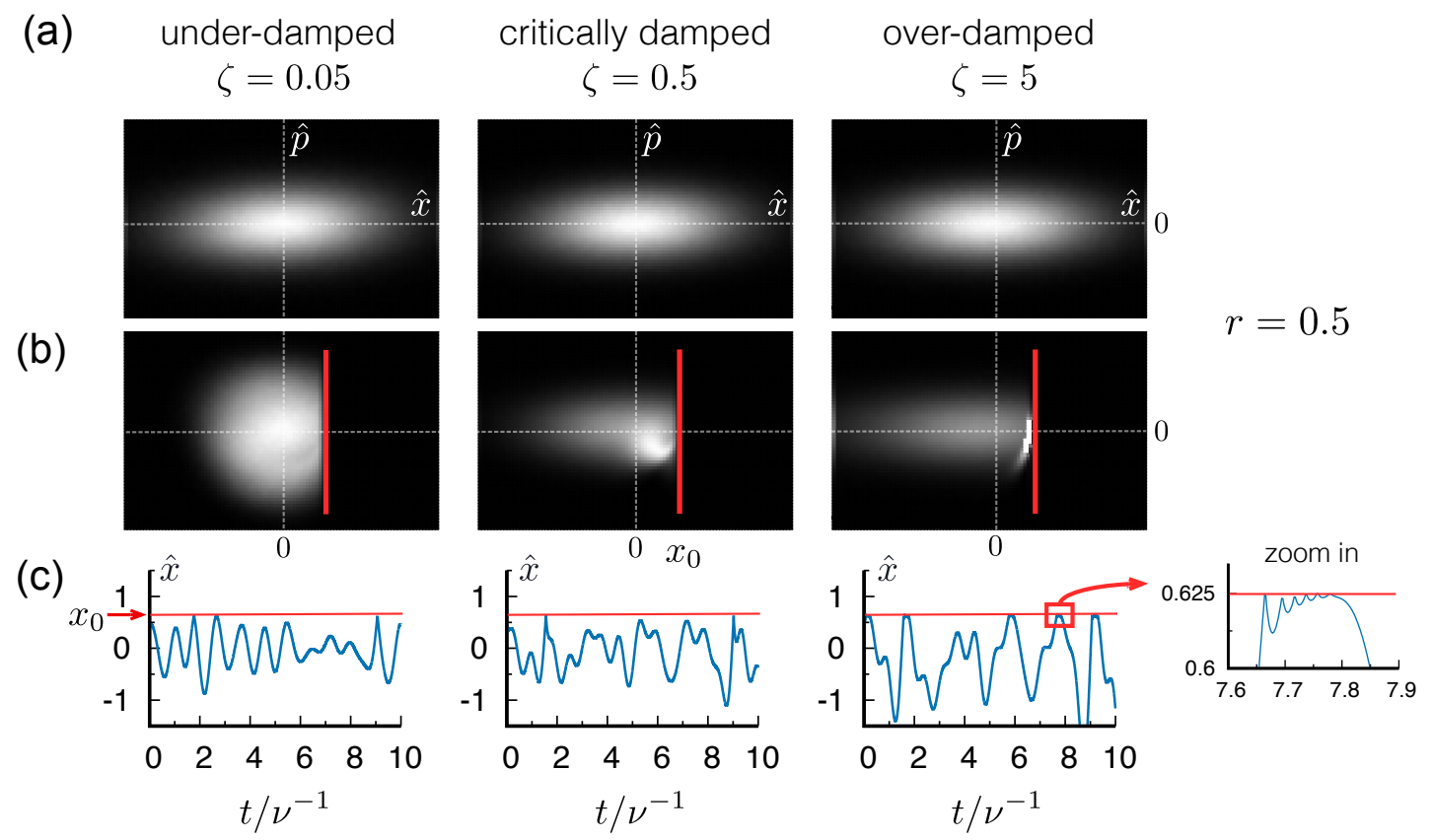

Figure 2. (a) Stroboscopic phase space densities of a harmonically confined particle subject to squeezed thermal noise for three different damping regimes: under-damped motion (damping ratio $\zeta=c / 2 m \omega_{0}=0.05$ ), critically damped motion $(\zeta=0.5$ ), and over-damped motion $(\zeta=5)$. The diagrams represent position-momentum histograms at equidistant points in time $t_{0}, \nu^{-1}+t_{0}, 2 \nu^{-1}+t_{0}, \ldots$, where $t_{0}=0.24 \nu^{-1}$ sets the relative timing of the observations with respect to the stochastic force. (b) Collisions with the piston (indicated by the red bar) induce phase jumps in the particle motion, which cancel the squeezing effect in the case of under-damped motion. In the case of over-damped motion, the particle tends to 'stick' to the piston, which leads to a strong enhancement of the probability density in this region. (c) Typical trajectories $x(t)$ of the trapped particle.

essentially unchanged. Note that the proposed scheme relies on the notion of a spatially compressed squeezed thermal state. We will first discuss some subtleties and apparent difficulties associated to the latter.

Figures 2 a,b show numerically obtained phase space densities of a confined single-particle gas subject to squeezed thermal noise in the under-damped (damping ratio $\left.\zeta=c / 2 m \omega_{0}=0.05\right)$, critically damped $(\zeta=0.5)$, and over-damped regime $(\zeta=5)$. For purely harmonic confinement (Fig. 2a), the response of the gas to the squeezed noise is largely independent of the damping regime. This is markedly different in the presence of a piston (Fig. 2b). Collisions of the particle with the piston induce phase-shifts in the otherwise purely harmonic motion. In the under-damped regime, these phaseshifts destroy the correlation between particle motion and squeezed noise, which cancels the squeezing phenomenon. In the critically damped and over-damped case, the collisions with the piston perturb, but do not destroy the squeezing phenomenon. In the over-damped region, an additional effect comes into play, namely, that the particle tends to 'stick' to the piston, which leads to a strong enhancement of the probability density in this region. This effect can also be observed in Fig. 22, which shows typical examples of the particle motion $x(t)$ in the various damping regimes. In the over-damped case, the particle tends to collide several times with the piston before it is finally accelerated in the opposite direction.

By recording the elastic collision events in our numerical simulations, we can derive the work $W$ required to compress the gas to half of its initial volume. In Fig. $3 \mathrm{a}$, $W$ is shown as a function of the parameter $t_{0}$, which defines the points in time, namely $t_{0}, \nu^{-1}+t_{0}, 2 \nu^{-1}+t_{0}, \ldots$, at which the compression steps are executed. At a relative timing around $t_{0}=0.33 \nu^{-1}$ and $t_{0}=0.83 \nu^{-1}$, the work $W$ is found to exponentially decrease with the squeezing parameter $r$ (note the logarithmic scale in Fig. 3a). Under those conditions, the squeezing effect reduces the occurrence of large positive momenta close to the piston (indicated by the red bar), which causes a reduced pressure exerted on the piston. Our numerical results, thus, give clear evidence that squeezing can be exploited to reduce the required work for the reset of a one-bit memory. Note that this effect applies to both the critical and the over-damped regime, but vanishes for strongly under-damped systems as shown in Fig. 3 b.

In the remainder of this work, we discuss a simplifying analytical model that captures the key aspects of the described phenomenon. The presence of a piston at position $x_{0}$ introduces a cut-off in phase space: $\rho\left(\hat{x}>x_{0}, \hat{p}\right)=0$. We will consequently model a spatially compressed squeezed thermal state by the density

$$
\rho(\hat{x}, \hat{p})=Z^{-1} \rho_{\mathrm{sq}}(\hat{x}, \hat{p}) \Theta\left(x_{0}-\hat{x}\right)
$$


(a)
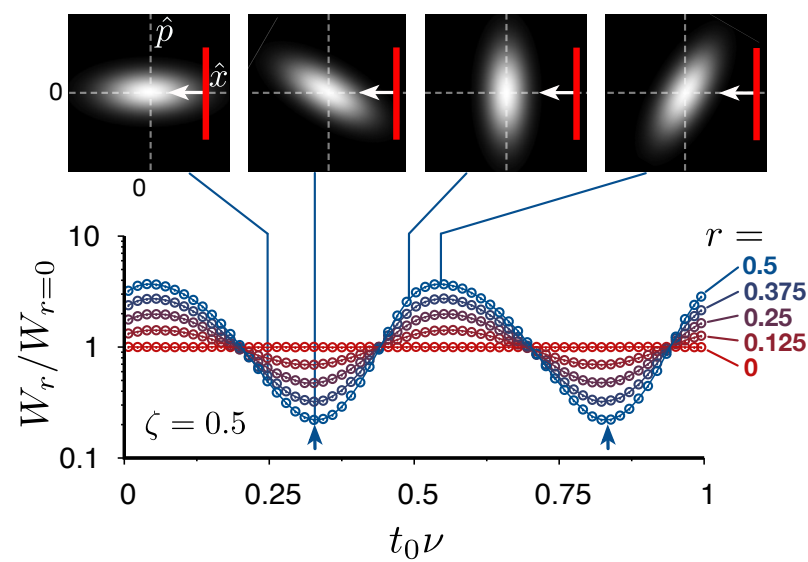

(b)

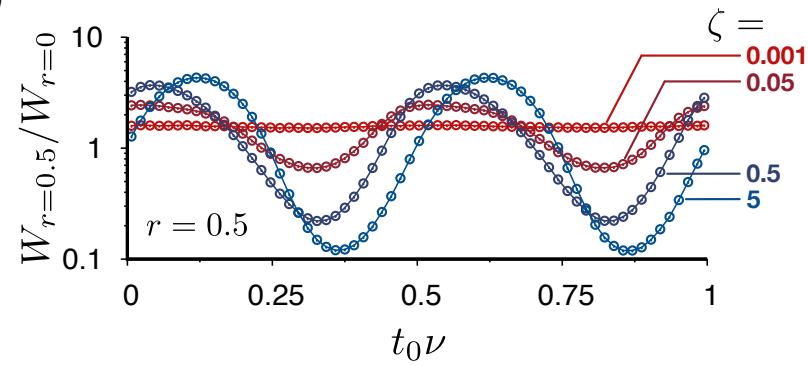

Figure 3. Required work $W$ to compress the single-particle gas to half of its initial volume as a function of the relative timing $t_{0}$, which defines the points in time, namely $t_{0}, \nu^{-1}+$ $t_{0}, 2 \nu^{-1}+t_{0}, \ldots$, at which the compression steps are executed. The given values of $W_{r}$ (for different squeezing parameters $r$ ) are normalized to the work at vanishing squeezing $W_{r=0}$. The temperature $T$ is kept fixed in all simulations. (a) In the critically damped regime $(\zeta=0.5)$, the work is observed to exponentially decrease with the squeezing parameter $r$ close to $t_{0}=0.33 \nu^{-1}$ and $t_{0}=0.83 \nu^{-1}$. (b) Work at constant squeezing $r=0.5$ for various damping ratios $\zeta$. The squeezing effect is observed to vanish for under-damped systems.

in which $\Theta(x)$ is the Heaviside step function $(\Theta(x)=1$ for $x>0, \Theta(x)=0$ otherwise) and $Z$ is a normalization constant such that $\iint \rho(\hat{x}, \hat{p}) d \hat{x} d \hat{p}=1$. We choose to perform the compression step against a purely momentum squeezed state of the gas as depicted in Fig. 2 a. To this end, we set $T_{x}=T \exp (+2 r)$ and $T_{p}=T \exp (-2 r)$ [19]. To derive the work for the compression, we start with a common ansatz in the kinetic gas theory relating the pressure exerted on the piston to the average momentum transfer by elastic collisions: $P=\int_{0}^{\infty} 2 \hbar \omega \hat{p}^{2} \rho\left(x_{0}, \hat{p}\right) d \hat{p}$. Using eq. (4), this results in

$$
P=2 b_{0} g\left(b_{0}\right) k_{\mathrm{B}} T_{p} / x_{0},
$$

in which we have introduced $b_{0}=\sqrt{\hbar \omega x_{0}^{2} / 2 k_{\mathrm{B}} T_{x}}$ and the auxiliary function $g(x)=\pi^{-1 / 2} \exp \left(-x^{2}\right) /(\operatorname{erf}(x)+1)$. With this, the required work $W=\int_{\infty}^{0} P d x_{0}$ follows as

$$
W=\ln 2 k_{\mathrm{B}} T_{p}=\ln 2 k_{\mathrm{B}} T \mathrm{e}^{-2 r} .
$$

This result confirms the exponential decrease of $W$ with increasing $r$, as observed in the numerical simulations.
There is, however, a certain discrepancy regarding the numerical pre-factor in the exponential scaling, see Ref. [30] for further details.

Since the probability density in eq. (4) factorizes as $\rho(\hat{x}, \hat{p})=\rho(\hat{x}) \rho(\hat{p})$ with $\rho(\hat{x})=\int_{-\infty}^{+\infty} \rho(\hat{x}, \hat{p}) d \hat{p}$ and $\rho(\hat{p})=\int_{-\infty}^{+\infty} \rho(\hat{x}, \hat{p}) d \hat{x}$, the entropy of a squeezed thermal state results additively from the contributions of the two quadratures: $S=S_{x}+S_{p}$. This is quite analogous to the well known additivity of entropy for independent subsystems. The two contributions can be determined using the Shannon entropy, which coincides with the physical entropy in the case of Gibbs ensembles. From $S_{p}=-k_{\mathrm{B}} \int_{-\infty}^{+\infty} \rho(\hat{p}) \ln (\rho(\hat{p})) d \hat{p}$, one concludes that the entropy in the momentum quadrature follows as

$$
S_{p} / k_{\mathrm{B}}=\ln \left(k_{\mathrm{B}} T_{p} / \hbar \omega\right) / 2+C .
$$

with an additive constant $C$. Note that this result does not reflect the correct low-temperature behavior of the entropy, which is an artifact of the purely classical calculation. This is, however, not crucial for the purpose of this work. In the same way, we derive a corresponding expression for the entropy in the position quadrature

$$
S_{x} / k_{\mathrm{B}}=\ln \left[\frac{x_{0}}{b_{0} g\left(b_{0}\right)}\right]-b_{0} g\left(b_{0}\right)-b_{0}^{2}+C^{\prime}
$$

During the free expansion (step $\mathrm{i}$ in Fig. 1) no work is performed. The internal energy $U=$ $\iint d \hat{x} d \hat{p} \rho(\hat{x}, \hat{p})(\hbar \omega / 2)\left(\hat{x}^{2}+\hat{p}^{2}\right)$, which using eq. 44 evaluates to

$$
U=\frac{k_{B}}{2}\left[\left(1-2 b_{0} g\left(b_{0}\right)\right) T_{x}+T_{p}\right],
$$

remains constant: $U\left(T_{x}, T_{p}, x_{0}=\infty\right)=U\left(T_{x}, T_{p}, x_{0}=0\right)$. Consequently, there is no net heat flow between system and environment and the entropy of the environment remains constant: $(\Delta S)_{\mathrm{env}}=0$. The total entropy change $\Delta S=(\Delta S)_{\mathrm{env}}+(\Delta S)_{\mathrm{sys}}$ is solely determined by the entropy change of the system $(\Delta S)_{\mathrm{sys}}=\Delta S_{x}+\Delta S_{p}$, which here is given by $(\Delta S)_{\mathrm{sys}}=S_{x}\left(x_{0}=\infty\right)-S_{x}\left(x_{0}=0\right)$. With eq. (8), this leads to a total entropy change of

$$
\Delta S=k_{\mathrm{B}} \ln 2 .
$$

This is the expected result for an irreversible doubling of the phase space volume. During the isothermal compression (step ii in Fig. 1) the invested work $W$ is dissipated as heat, which leads to an entropy increase in the environment of $(\Delta S)_{\mathrm{env}}=W / T_{p}=k_{\mathrm{B}} \ln 2$ that exactly cancels the entropy decrease in the system $(\Delta S)_{\mathrm{sys}}=-k_{\mathrm{B}} \ln 2$. Thus, no net change in the total entropy occurs during this step. The same is obviously true for the third and last step, the insertion of the partition. This means that the total entropy change of the universe during the erasure process solely results from the entropy increase during the free expansion and is consequently given by eq. (10). In total, we find that the reset of one bit of classical information in a squeezed thermal memory leads to the 
same entropy increase of $k_{\mathrm{B}} \ln 2$ as in a standard thermal memory, while the required work can be exponentially lowered with the squeezing factor. We expect that an experimental verification of the predicted effect using well-established experimental platforms such as optically trapped nano-particles [7, 8, 31] and nano-mechanical devices [27] is within reach.

Squeezed thermal environments are characterized by fast periodic amplitude modulations in the thermal fluctuations. The significance of such non-equilibrium thermal reservoirs stems from the fact that they may naturally arise in systems operating in a pulse-driven fashion as is common, for example, in digital electronics. The dissipated power in today's microprocessors is due to both static leakage and dynamic power dissipation, in approximately equal parts [2]. The dynamic power dissipation in a CPU originates from the switching of logic gates. The latter is physically realized by charging or discharging capacitors within the gate. This process is accompanied by current flows and associated ohmic losses. If the gate switches periodically in time, it thus acts as a periodic heat source. As an approximation, one can consider a gate as a point-like heat source that periodically dissipates energy with frequency $\Omega$ in a material with heat diffusivity $\alpha_{1}$. In this situation, fast periodic modulations of the temperature arise that spatially extent into the environment 32 . Such a transient temperature phe- nomenon is nothing but a squeezed thermal environment, which can be seen by comparison with eq. (2). The spatial extent of this environment can be estimated as several times the characteristic decay length $\sqrt{\alpha_{1} / \pi \Omega}[32$, which for $\Omega=1 \mathrm{GHz}$ and the thermal diffusivity of silicon corresponds to several hundred nanometers. Thus, the periodic power dissipation in a logic gate induces a squeezed thermal bath in its surroundings that may even affect neighboring gates. The magnitude of this effect, the squeezing factor, depends on a multitude of factors such as geometry, thermal conductivity of materials and thermal resistance of interfaces. Using advanced design approaches, such as thermal rectification [33, thermal flows can even be decoupled from electronic currents, which further expands the possibilities to deliberately engineer thermal environments. Similar to what has been demonstrated in this Letter, a well-timed switching process may exploit transient temperature phenomena to reduce the overall dissipated power. The latter applies to all systems in which the energy costs depend on the temperature - even if they operate well above the Landauer limit. In future, combining concepts of electronics and non-equilibrium thermodynamics will open up new routes for more energy efficient electronics.

We thank Emre Togan, Atac Imamoglu, and Willem Vos for fruitful discussions.
[1] M. P. Frank, Comput. Sci. Eng. 4, 16 (2002).

[2] W. Haensch et al., IBM J. Res. Dev. 50, 339 (2006).

[3] E. Pop, Nano Res. 3, 147 (2010).

[4] R. Landauer, IBM J. Res. Develop. 5, 183 (1961).

[5] M. B. Plenio and V. Vitelli, Contemp. Phys. 42, 25 (2001).

[6] J. A. Vaccaro and S.M. Barnett, Proc. R. Soc. A 467, 1770 (2011).

[7] A. Berut, A. Arakelyan, A. Petrosyan, S. Ciliberto, R. Dillenschneider, and E. Lutz, Nature 483, 187 (2012).

[8] Y. Jun, M. Gavrilov, and J. Bechhoefer, Phys. Rev. Lett. 113, 190601 (2014).

[9] J. M. R. Parrondo, J.M. Horowitz, and T. Sagawa, Nat. Phys. 11, 131 (2015)

[10] J. Hong, B. Lambson, S. Dhuey, and J. Bokor, Sci. Adv. 2, e1501492 (2016).

[11] L. L. Yan, T. P. Xiong, K. Rehan, F. Zhou, D. F. Liang, L. Chen, J. Q. Zhang, W. L. Yang, Z. H. Ma, and M. Feng, Phys. Rev. Lett. 120, 210601 (2018).

[12] S. Hilt, S. Shabbir, J. Anders, and E. Lutz, Phys. Rev. E 83, 030102(R) (2011).

[13] L. del Rio, J. Aberg, R. Renner, O. Dahlsten, and V. Vedral, Nature 476, 476 (2011).

[14] M. Esposito and C. van den Broeck, Eur. Phys. Lett. 95, 40004 (2011).

[15] J. Goold, M. Paternostro, and K. Modi, Phys. Rev. Lett. 114, 060602 (2015).

[16] M. Pezzutto, M. Paternostro, and Y. Omar, New J. Phys. 18, 123018 (2016).

[17] J. Millen and A. Xuereb, New J. Phys. 18, 011002 (2016).
[18] G. Manzano, arXiv:1711.07817 (2017).

[19] H. Fearn and M. J. Collett, J. Mod. Opt. 35, 553 (1988).

[20] M. S. Kim, F. A. M. de Oliveira, and P. L. Knight, Phys. Rev. A 40, 2494 (1989).

[21] D. Rugar and P. Grutter, Phys. Rev. Lett. 67, 699 (1991).

[22] J. Rossnagel, O. Abah, F. Schmidt-Kaler, K. Singer, and E. Lutz, Phys. Rev. Lett. 112, 030602 (2014).

[23] L. A. Correa, J. P. Palao, D. Alonso, and G. Adesso, Sci. Rep. 4, 3949 (2014).

[24] G. Manzano, F. Galve, R. Zambrini, and J. M. R. Parrondo, Phys. Rev. E 93, 052120 (2016).

[25] W. Niedenzu, D. Gelbwaser-Klimovsky, A. G. Kofman, and G. Kurizki, New J. Phys. 18, 083012 (2016).

[26] W. Niedenzu, V. Mukherjee, A. Ghosh, A.G. Kofman and G. Kurizki, Nat. Commun., doi: 10.1038/s41467017-01991-6 (2018).

[27] J. Klaers, S. Faelt, A. Imamoglu, and E. Togan, Phys. Rev. X 7, 031044 (2017).

[28] M. O. Scully, Phys. Rev. Lett. 87, 220601 (2001).

[29] G. Manzano, arXiv:1806.07448 (2018).

[30] See Supplemental Material at [URL] for further information.

[31] M. Rashid, T. Tufarelli, J. Bateman, J. Vovrosh, D. Hempston, M. S. Kim, and H. Ulbricht, Phys. Rev. Lett. 117, 273601 (2016).

[32] P. Michaud, Y. Sazeides, A. Seznec, T. Constantinou, D. Fetis, RR-5744, INRIA. 2005, pp. 32.

[33] N. A. Roberts and D. G. Walker, Int. J. Therm. Sci. 50, 648 (2011). 